\title{
Influence du télétriage médical assisté par ordinateur sur le comportement des patients: premières expériences en Suisse
}

\author{
A. Meer ${ }^{a}$, C. Simonin ${ }^{a}$, A. Trapp ${ }^{a}$, S. Niemann ${ }^{b}$, T. Abel ${ }^{b}$
}

Deutsch erschienen

in Nr. 41/2003

\footnotetext{
a Medvantis medi-a4

b Institut de médecine sociale et préventive de l'Université de Berne, Département de recherche de santé
}

Correspondance:

Dr A. Meer

Medvantis medi-24

Bolligenstrasse 52

CH-3006 Berne

Tél. 0313400500

Fax 0313400555

E-mail: a.meer@medvantis.ch

\section{Synthèse}

Depuis le printemps 2000, la société Medvantis medi-24 propose en Suisse un télétriage médical assisté par ordinateur. L'évaluation scientifique de ce nouveau service fait partie intégrante du processus d'assurance qualité. C'est pourquoi Medvantis medi-24 a chargé l'Institut de médecine sociale et préventive (IMSP) de l'Université de Berne de réaliser cette étude.

En ce qui concerne les mesures à prendre et leur degré d'urgence, 70\% des appelants avaient une appréciation de leur problème divergeant de celle du spécialiste du centre d'expertise médicale. Après l'entretien, $63 \%$ des personnes interrogées ont modifié leur intention première. Les médecins ayant traité les appelants ultérieurement ont répondu à un questionnaire écrit et plus de 90\% d'entre eux ont estimé que les consultations recommandées par le centre d'expertise médicale, tout comme le délai conseillé pour se rendre chez le médecin, étaient justifiés.

L'étude révèle que le grand public n'est généralement pas capable d'évaluer correctement la gravité de la situation et qu'il existe dans ce domaine un manque d'information. Le degré relativement élevé d'application des conseils fournis par le centre d'expertise médicale souligne l'efficacité de ce service et montre que ce nouvel instrument de la santé suisse exerce une influence sur le comportement du patient. La méthode d'évaluation de la qualité de l'aiguillage doit encore être perfectionnée. Nous supposons que ce service joue un rôle important dans la réduction des coûts des soins de santé.

\section{Introduction}

Depuis le printemps 2000, la société Medvantis medi-24 propose les services d'un centre d'expertise médicale en Suisse. Son but est de fournir aux patients des réponses précises et objectives à leurs questions d'ordre médical et des conseils sur la meilleure façon d'agir. Les conseillères de santé sont assistées par un système expert médical lors du questionnement structuré et orienté symptômes ainsi que lors de l'aiguillage. Sur la base des connaissances médicales des conseillères de santé et des médecins et en fonction du résultat fourni par le système informatique, les appelants reçoivent l'une des instructions reprises dans le tableau 2 .

Il importe de contrôler l'efficacité et la qualité des services existants et nouveaux en matière médicale. C'est la raison pour laquelle Medvantis Suisse ne s'est pas contentée des contrôles de qualité internes et a chargé l'Institut de médecine sociale et préventive de l'Université de Berne d'évaluer ses services de manière scientifique dans le cadre d'un processus permanent. La présente étude apporte les premiers résultats.

Tableau 1

Conclusions essentielles.

En ce qui concerne les mesures à prendre et leur degré d'urgence, $70 \%$ des appelants avaient une appréciation de leur problème divergeant de celle du spécialiste du centre d'expertise médicale.

- $63 \%$ des personnes interrogées ont modifié leur intention première à la suite de l'entretien téléphonique avec la conseillère de santé.

- Seuls $2 \%$ des appelants ont vu leur intention de se rendre d'urgence à l'hôpital confirmée.

- $81 \%$ des appelants ayant la même intention ont changé d'avis après l'entretien et ont pu se soigner eux-mêmes ou l'être par le médecin de famille ou de garde.

- 90\% des médecins interrogés ont estimé que la consultation recommandée par le centre d'expertise médicale, tout comme le délai conseillé pour celle-ci, étaient justifiés.

- Le grand public n'est généralement pas capable d'évaluer correctement la gravité du problème médical. 
Tableau 2

Niveaux d'aiguillage, aiguillage et instructions.

\begin{tabular}{lll}
$\begin{array}{l}\text { Niveau } \\
\text { d'aiguillage }\end{array}$ & Aiguillage & Instructions \\
\hline 1 & A l'hôpital d'urgence $(\mathrm{HU})$ & $\begin{array}{l}\text { Aiguiller sur les services d'urgence régionaux } \\
144 \text { et préconiser l'hospitalisation. }\end{array}$ \\
\hline 2 & Consultation immédiate $\mathrm{Cl})$ & $\begin{array}{l}\text { Recommander une consultation immédiate } \\
\text { chez le médecin de famille ou celui de garde. }\end{array}$ \\
\hline 3 & $\begin{array}{l}\text { Visite chez le médecin } \\
\text { dans les } 24 \text { heures (CMO) }\end{array}$ & $\begin{array}{l}\text { Conseiller une visite chez le médecin de } \\
\text { famille ou celui de garde dans les } 24 \text { heures. }\end{array}$ \\
\hline 5 & Consultation de routine (CMO) & $\begin{array}{l}\text { Suggérer une consultation chez le médecin } \\
\text { de famille dans les } 2 \text { à } 7 \text { jours suivants. }\end{array}$ \\
\hline 4 & Autotraitement (AT) & $\begin{array}{l}\text { Pas de consultation. La conseillère de santé } \\
\text { donne des instructions pour l'autotraitement. }\end{array}$
\end{tabular}

\section{Matériel et méthodes}

Du 26 février 2001 au 30 juin 2001 et du 15 octobre 2001 au 5 février 2002, il a été demandé au début de l'entretien à plus de 2000 appelants ce qu'ils comptaient faire pour soigner leurs maux et quel était, selon eux, le degré d'urgence. L'intention a ensuite été comparée avec l'appréciation de la conseillère de santé à la fin du télétriage assisté par ordinateur. Avant la fin de l'entretien, on a demandé aux appelants s'ils acceptaient d'être sondés ultérieurement par l'IMSP. Sept à dix jours après le contact avec le centre d'expertise médicale, les collaborateurs de l'IMSP ont interrogé par téléphone la population étudiée sur l'efficacité du conseil ainsi que sur le temps écoulé entre l'application de celui-ci et l'entretien téléphonique. Toutes les questions étaient ouvertes et les réponses ont été classées en fonction de treize intentions possibles et de 6 degrés d'urgence. La formulation ouverte a été choisie, car elle permettait d'éviter au maximum que la question ne biaise la réponse. Les 78 combinaisons de réponses différentes ont été attribuées via une matrice aux cinq niveaux d'aiguillage du centre d'expertise médicale. De plus, un sixième groupe d'aiguillage a été défini («groupe d'aiguillage 6»). Celui-ci concerne les appelants qui ont clairement indiqué que leur intention se fondait sur un entretien téléphonique médical fiable. Avant la collecte des données, la matrice a été établie sur la base d'une réunion des représentants médicaux et paramédicaux de l'IMSP et du centre d'expertise médicale.

Les trois combinaisons - intention/urgence, résultat de l'aiguillage/urgence et les mesures réellement prises et le temps écoulé entre celleci et le contact avec le centre d'expertise médicale - ont été comparées entre elles et examinées à la lumière des deux critères d'évaluation suivants:

1. divergence entre l'intention/l'urgence et l'instruction du centre d'expertise médicale; 2. application du conseil fourni par le centre d'expertise médicale (compliance).

Les données de l'étude ont été dépouillées selon des méthodes statistiques descriptives et inférentielles. Dans cette publication, les données proviennent principalement de la statistique descriptive. Les valeurs de signification apparaissent en contexte dans la section Discussion et se fondent sur le test de McNemar. Le $\mathrm{khi}^{2}$ de McNemar se calcule comme suit: $\mathrm{khi}^{2}=$ $(b-c)^{2} / b+c(d f=1)$.

Les variables dichotomiques (degré de liberté $\mathrm{df}=1$ ) des intentions et de l'aiguillage ou, selon le cas, des intentions et du comportement sont représentées dans un tableau à quatre entrées. Le test McNemar compare les cas de correction à la hausse ou à la baisse de $\mathrm{b}$ et $\mathrm{c}$ (off diagonale) et contrôle leur signification. On vérifie l'hypothèse nulle, selon laquelle les proportions du passage de $\mathrm{b}$ à $\mathrm{c}\left(\mathrm{p}_{\mathrm{b}}^{\prime}\right)$ et inversement $\left(\mathrm{p}_{\mathrm{c}}^{\prime}\right)$ sont égales: $p^{\prime}=p_{b}^{\prime}=p_{c}^{\prime}=\left(p_{b}^{\prime}+p_{c}^{\prime}\right) / 2$.

Medvantis Suisse emploie quatre médecins spécialistes et dix-huit employés médicaux qualifiés disposant d'une solide formation de base en soins de santé ainsi que d'une formation interne supplémentaire sur l'activité de conseil dans un centre d'expertise médicale. L'expérience professionnelle pratique moyenne des conseillères de santé est de dix ans. Le processus de conseil ainsi que sa consignation sont assurés par un système informatique (TAS/Plain Software Company/UK).

La seconde phase de collecte des données a consisté à interroger par écrit les médecins ayant traité les appelants ultérieurement. Pendant l'interview par l'IMSP, il a été demandé aux appelants qui avaient entre-temps consulté un médecin s'ils acceptaient que ce dernier soit également intégré à l'étude. Les praticiens ont alors déterminé si la consultation survenue à la suite du contact avec le centre d'expertise médicale était justifiée et si le délai fixé pour celle-ci avait été correctement évalué. L'IMSP n'a pas été révélé aux médecins participant à l'évaluation l'aiguillage du centre d'expertise médicale.

\section{Résultats}

L'IMSP a pu contacter 1743 des 2092 appelants qui ont donné leur consentement pour être rappelés. 129 sujets collectés n'ont pu être 
Figure 1

Distribution de fréquences des intentions $(n=1614)$.

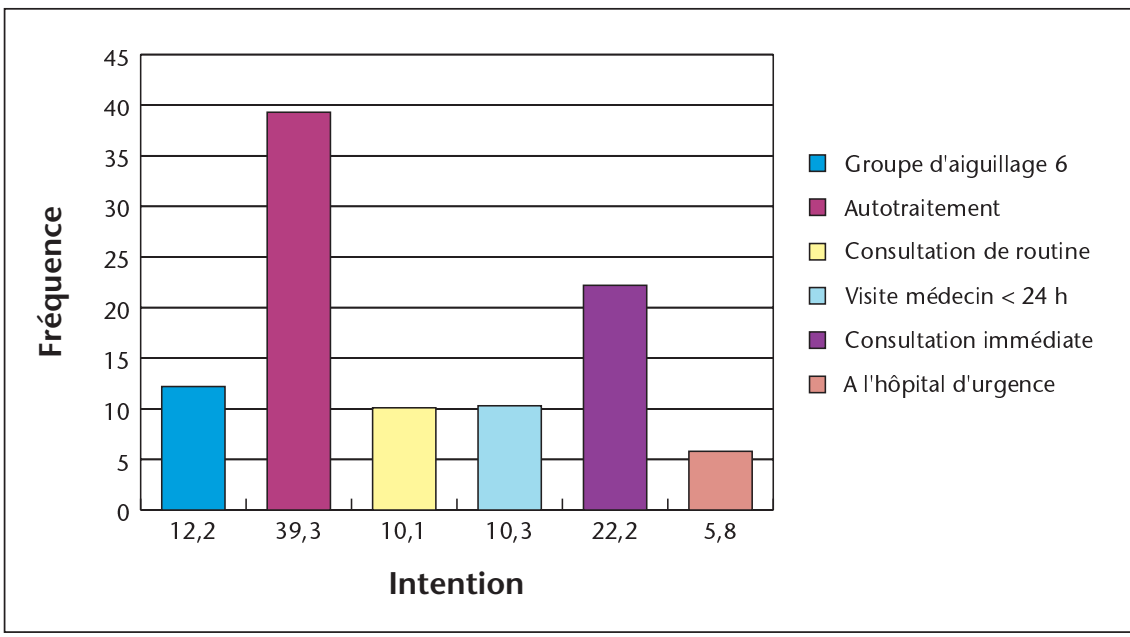

Figure 2

Distribution de fréquences de l'aiguillage $(n=1614)$.

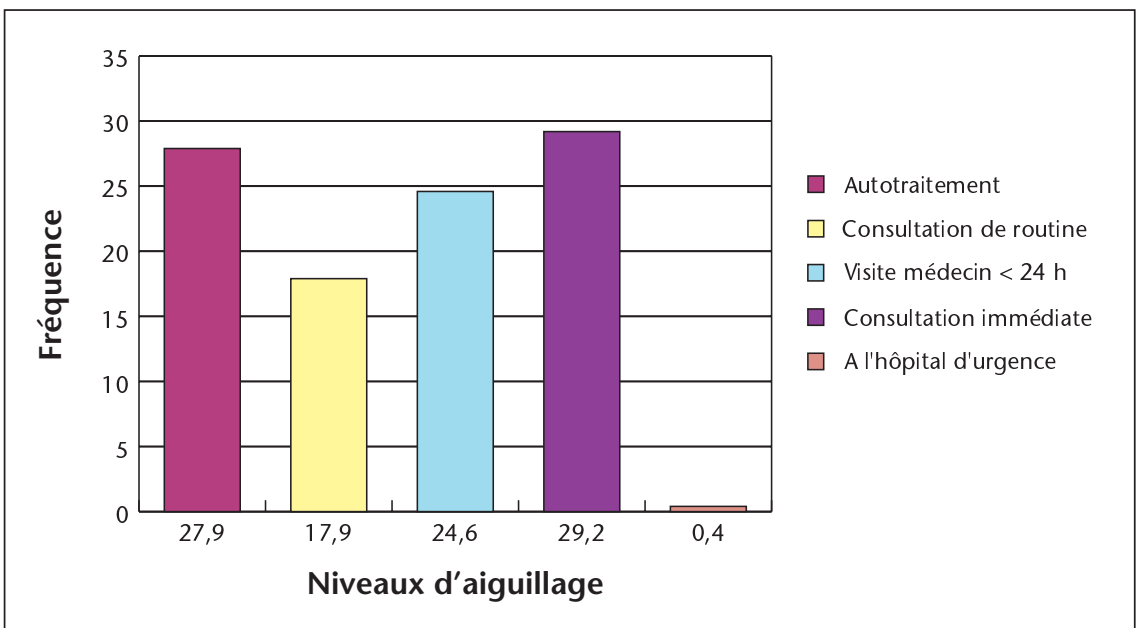

attribués aux 78 combinaisons de réponses et ont donc été exclus de l'étude. Sur les 1614 appels exploités, 197 (12\%) personnes interrogées n'avaient pas d'intention précise et ont déclaré qu'elles s'étaient fait conseiller par téléphone par un assistant médical qualifié. Elles ont été attribuées au «groupe d'aiguillage 6». Les distributions de fréquences, calculées en pour cent, des différents niveaux d'aiguillage figurent aux figures 1 à 3 .

Les confrontations intention/aiguillage, aiguillage/comportement et intention/comportement sont reprises dans les figures 4 à 6 . Les niveaux d'aiguillage «visite chez le médecin dans les 24 heures» et «consultation de routine» ont été agrégés à «consultation médicale ordinaire» (CMO). Les données correspondent ainsi mieux à une optique de réduction des coûts de soins de santé, car les distinctions entre consultation urgente chez un médecin ou à l'hôpital et consultation médicale ordinaire ou autotraitement prennent ici toute leur importance.

En ce qui concerne les mesures à prendre et leur degré d'urgence, 70\% des appelants avaient une appréciation de leur problème divergeant de celle du spécialiste du centre d'expertise médicale. A la suite de l'entretien téléphonique, $63 \%$ des personnes interrogées ont modifié leur intention première. Le degré d'application moyen de tous les niveaux d'aiguillage est de $53 \%$.

La comparaison entre l'intention avant le conseil fourni et le comportement après celui-ci a révélé au total 426 corrections à la baisse vers des niveaux de soins moins coûteux et 405 corrections à la hausse vers des niveaux plus onéreux. La fréquence relative des modifications de niveaux de soins est illustrée par le figure 7 . Les corrections à la baisse prédominent dans les soins d'urgence dispendieux (1-2: de à l'hôpital d'urgence à consultation immédiate, 1-3: de à l'hôpital d'urgence à visite chez le médecin dans les 24 heures, 1-4: de à l'hôpital d'urgence à consultation de routine, 1-5: de à l'hôpital d'urgence à autotraitement, 2-3: de consultation immédiate à visite chez le médecin dans les 24 heures, 2-5: de consultation de routine à autotraitement). En revanche, les corrections à la hausse les plus nombreuses concernaient la consultation de routine dans les 24 heures (3-4: consultation de routine à visite chez le médecin dans les 24 heures, 3-5: autotraitement à visite chez le médecin dans les 24 heures).

291 appelants ont accepté que le médecin qui les a soignés par la suite soit interrogé sur la consultation. 261 médecins ont été contactés par courrier et 205 questionnaires ont pu être dépouillés (retour 79\%). Dans 90\% (185) des cas, la consultation a été considérée comme justifiée, 3\% (5) sont restés sans réponse et 7\% (15) des consultations ont été qualifiées de non fondées. Le moment des consultations jugées justifiées a été correctement évalué dans 91\% (153) des cas, il a été estimé précipité dans 5\% (10) et tardif dans $4 \%$ (7).

\section{Discussion}

Les systèmes de soins de santé des différents pays européens se distinguent par certains facteurs critiques qui influent sur le recours par les patients aux services d'un centre d'expertise médicale. Par exemple, la densité des cabinets 
Figure 3

Distribution de fréquences du comportement $(n=1614)$.

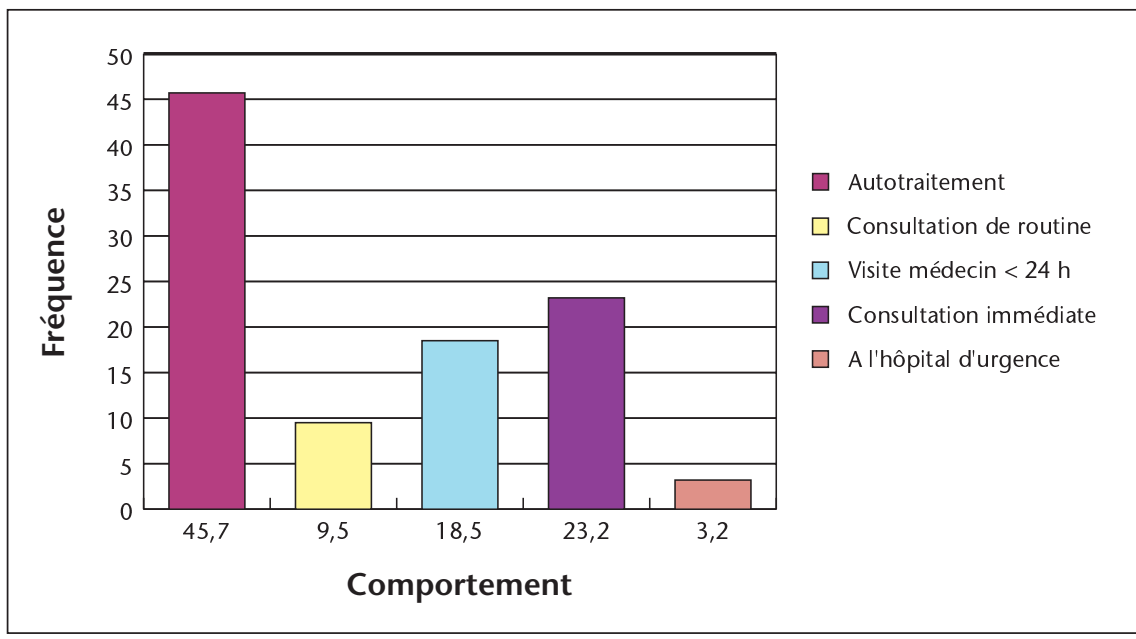

Figure 4

Ecart entre l'aiguillage et l'intention des appelants. Chiffres absolus des intentions entre parenthèses: $\mathrm{HU}=$ à I'hôpital d'urgence (93), Cl = consultation immédiate (359), CMO = consultation médicale ordinaire (330), AT = autotraitement (635), GA6 = groupe d'aiguillage 6 (197).

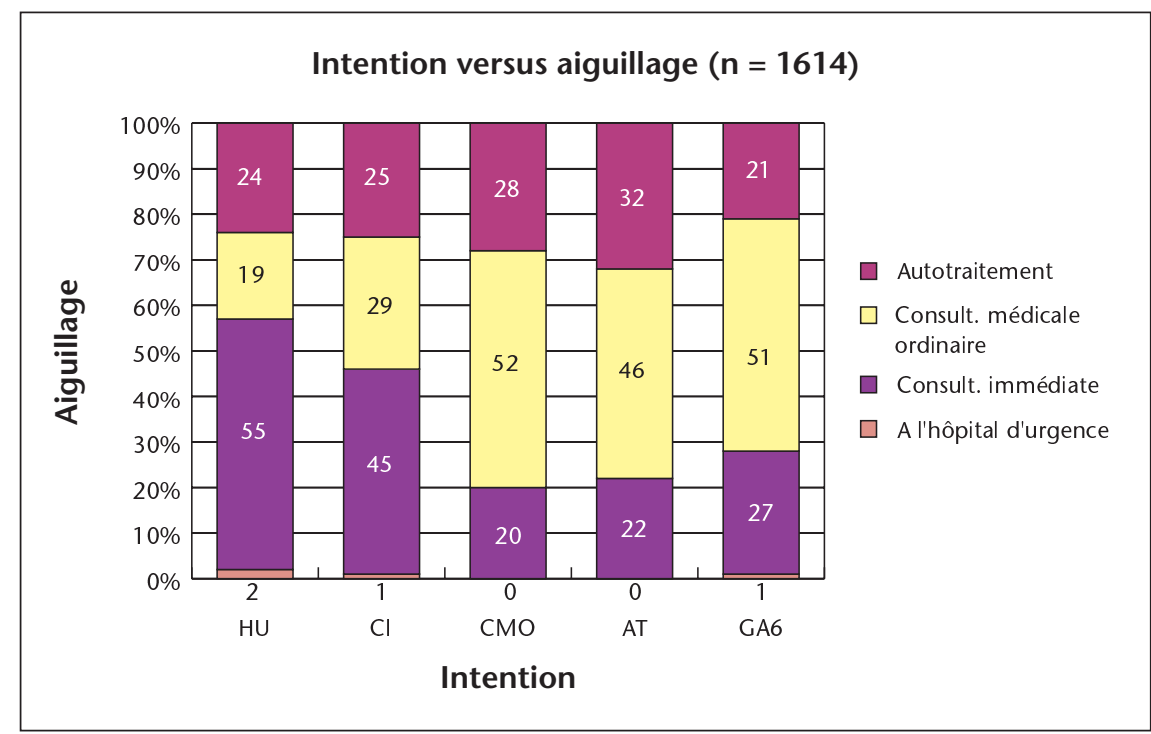

1 Munro J, Nicholl J, O'Cathain A, Knowles E. Evaluation of NHS Direct first wave sites: First interim report to the Department of Health. Sheffield: Medical Care Research Unit; 1998

2 Flaig C, Zehnder K, Zürcher $\mathrm{H}$ Eichenberger P, Frei C, Gegeckas A et al. Selbsteinweisungen ins Spital. PrimaryCare 2002;2:280-3. médicaux, la disponibilité du médecin traitant, l'organisation des services d'urgence et d'ambulance et le financement sont différents dans chaque pays. En outre, diverses institutions (assurances, associations de médecins, organes publiques) ayant un contexte et des objectifs différents proposent cette prestation aux patients. En raison de cette hétérogénéité, la possibilité d'effectuer une comparaison internationale et de transférer des résultats et expériences scientifiques liés à la prestation des centres d'expertise médicale est restreinte. De plus, l'évaluation scientifique fait partie intégrante du processus d'assurance qualité et devrait dès lors être réalisée pays par pays et en tenant compte de chaque situation spécifique.

Cette étude a comparé l'intention des appelants, l'aiguillage des conseillères de santé et le comportement des appelants.

En ce qui concerne les mesures à prendre et leur degré d'urgence, 70\% des appelants avaient une appréciation de leur problème divergeant de celle du spécialiste du centre d'expertise médicale. Munro et al. ont effectué une étude semblable dans le cadre de l'évaluation scientifique du NHS Direct au Royaume-Uni. Dans celle-ci, $52 \%$ des personnes interrogées avaient une intention différente du résultat de l'aiguillage [1]. Au moyen d'un questionnaire écrit, on leur a demandé, une à plusieurs semaines après le contact avec le centre d'expertise médicale, quelle avait été leur intention. Afin d'éviter que le conseil fourni ait une quelconque influence sur la réponse à cette question, nous avons choisi, pour notre étude, de poser celle-ci aux appelants au début de l'entretien téléphonique. L'apparente meilleure auto-estimation de la population britannique étudiée est probablement due à une erreur systématique engendrée par la méthode de sondage rétrospectif. Pour les niveaux d'aiguillage «à l'hôpital d'urgence» $(98 \%, \mathrm{p}<0,001)$, «consultation immédiate» $(55 \%, \mathrm{p}<0,001)$ et «autotraitement» $(68 \%, p<0,001)$, les écarts étaient particulièrement importants.

Cette étude révèle que le grand public n'est généralement pas capable d'évaluer correctement la gravité du problème médical. Il semble qu'il lui soit particulièrement difficile d'évaluer correctement les symptômes et de décider valablement, sur la base d'une symptomatologie existante, la possibilité d'un autotraitement. Les services d'un centre d'expertise médicale semblent dès lors pertinents.

Dans l'ensemble, $63 \%$ des personnes interrogées ont modifié leur intention première après l'entretien téléphonique avec le centre d'expertise médicale. La conformité entre le conseil et l'action pour l'urgence et l'autotraitement était particulièrement élevée. Selon l'étude de Flaig et al. [2] menée dans deux hôpitaux suisses (hôpital cantonal de Baden, hôpital de district de Brougg), 80\% des patients qui se sont directement présentés à l'hôpital auraient pu être traités par un généraliste. La subjectivité de l'urgence, l'absence du médecin de famille, la proximité de l'hôpital et la méconnaissance des services d'urgence ont été avancées comme principales raisons ayant motivé les patients à se rendre directement à l'hôpital sans contacter auparavant leur médecin de famille ou de garde. 
Figure 5

Ecart entre le comportement des appelants et leur intention. Chiffres absolus des intentions entre parenthèses: $\mathrm{HU}$ = à l'hôpital d'urgence (93), Cl = consultation immédiate (359), CMO = consultation médicale d'urgence (330), AT = autotraitement (635), GA6 = groupe d'aiguillage 6 (197).

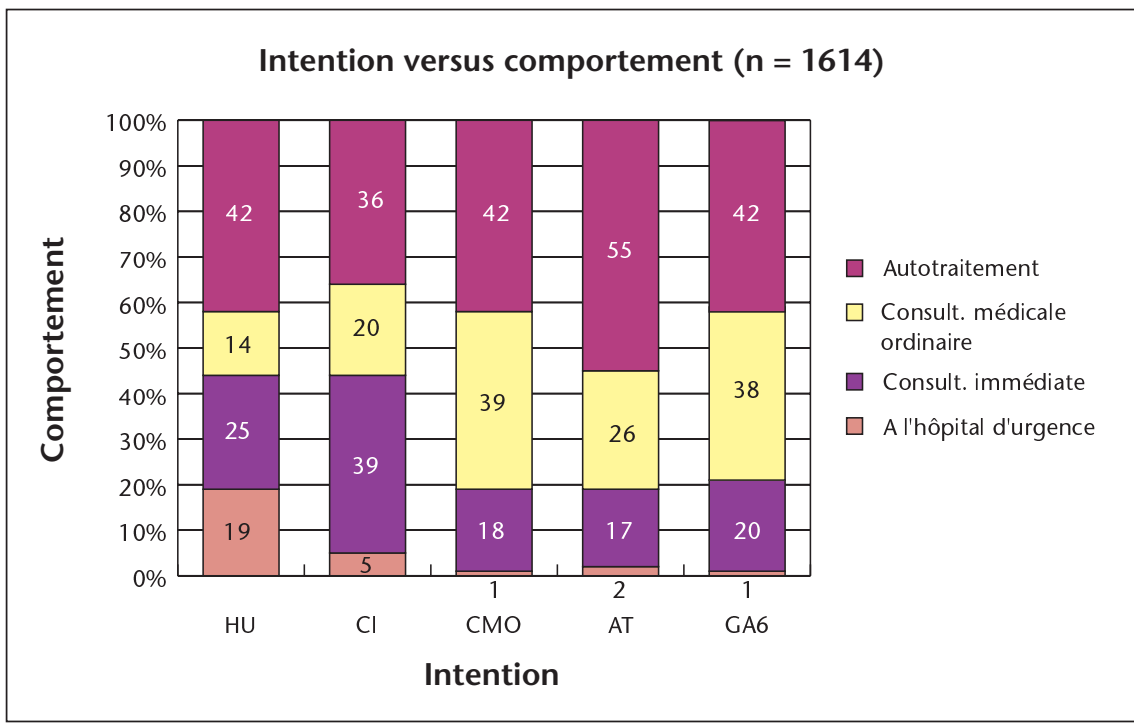

Figure 6

Ecart entre le comportement des appelants et le résultat de l'aiguillage. Chiffres absolus de I'aiguillage entre parenthèses: $\mathrm{HU}=$ à I'hôpital d'urgence (6), $\mathrm{Cl}$ = consultation immédiate (472), $\mathrm{CMO}=$ consultation médicale ordinaire (686), AT = autotraitement (450).

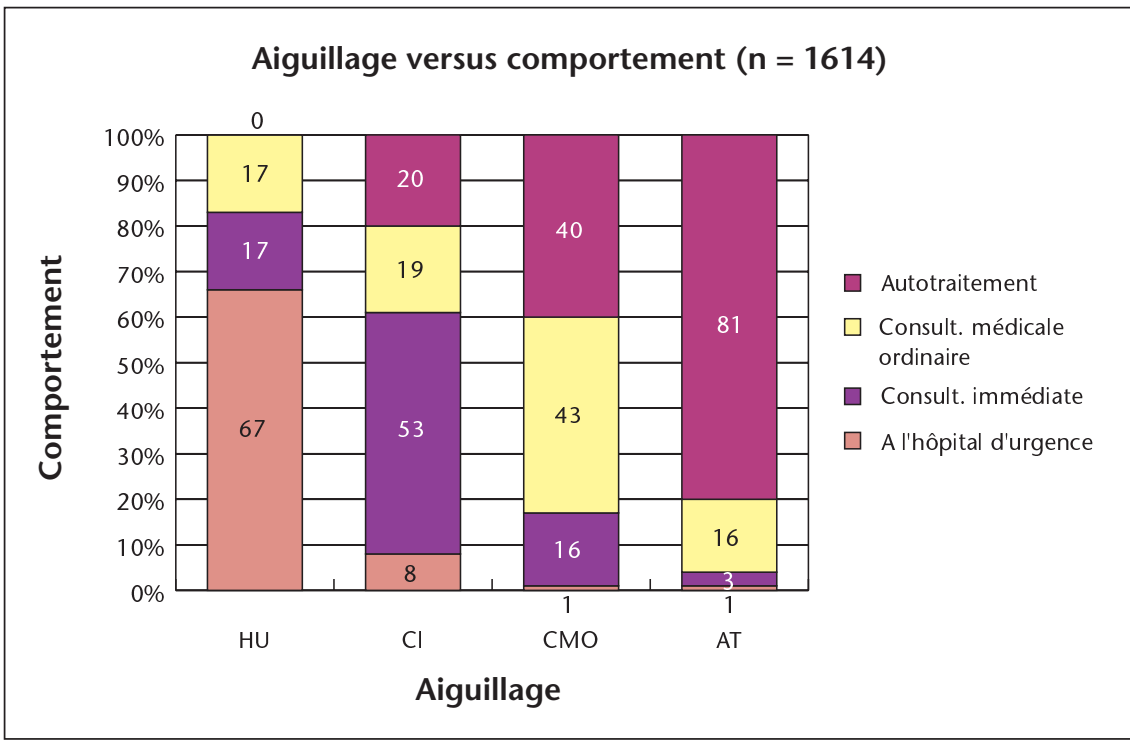

3 Munro J, Nicholl J, O'Cathain A, Knowles E, Morgan A. Evaluation of NHS Direct first wave sites:

Final report of the phase 1 research. Sheffield: Medical Care Research Unit; 2001.
Les données de cette étude confirment l'observation faite ci-dessus au sujet d'une utilisation inadéquate des ressources médicales par les patients. Seuls $2 \%$ des appelants ont vu la conseillère de santé confirmer leur intention de se rendre directement à l'hôpital. 81\% ( $\mathrm{p}<0,001)$ des appelants ayant la même intention ont changé d'avis après l'entretien et ont pu se soigner eux-mêmes ou l'être par leur médecin de famille ou celui de garde. L'activité de conseil d'un service d'expertise médicale semble efficace et permet une optimisation du processus ainsi qu'une meilleure allocation des ressources. La prestation d'un service d'expertise médicale peut juguler une surabondance et une insuffisance de soins de santé en Suisse.

La seconde phase de collecte des données a consisté à interroger par écrit les médecins ayant traité les appelants ultérieurement. Plus de 90\% d'entre eux ont estimé que la consultation, tout comme le délai conseillé pour celle-ci, étaient justifiés. Il s'agit en l'occurrence d'une collecte de données préliminaire effectuée dans la perspective d'élaborer un autre procédé d'évaluation de la prestation qui se baserait sur le jugement des médecins ayant traité les appelants. Ces informations sont purement descriptives et ne peuvent être utilisées directement comme instrument d'évaluation de la qualité de l'aiguillage.

Il n'est pas aisé d'apprécier la qualité des services médicaux ni la justesse d'un aiguillage médical. Dans le cadre des processus internes d'assurance qualité, Medvantis medi-24 fait évaluer les enregistrements des entretiens d'aiguillage ainsi que les recommandations qui en résultent par divers spécialistes (médecins indépendants, équipe médicale du centre d'expertise médicale, spécialistes médicaux nonmédecins). Le rapport final de la première phase d'étude du NHS Direct [3] souligne la forte variabilité interindividuelle de l'évaluation par les experts médicaux et suggère un processus plus complet d'assurance qualité. L'évaluation de la qualité devrait se fonder sur un panel d'experts possédant la plus grande expérience possible dans le domaine médical et les conseils résultant de l'aiguillage devraient être appréciés en fonction des informations recueillies lors de l'entretien et des données disponibles au moment de l'évaluation. En outre, il est recommandé d'analyser la sensibilité (p.ex. le nombre de patients qui avaient besoin d'une consultation en urgence et qui ont été aiguillés correctement) et la spécificité (p.ex. le nombre de patients qui n'avaient pas besoin de consultation en urgence et qui ont reçu une telle recomman- 
Figure 7

Les corrections à la baisse prédominent dans les soins d'urgence dispendieux (1-2: de à l'hôpital d'urgence à consultation immédiate, 1-3: de à l'hôpital d'urgence à visite chez le médecin dans les 24 heures, 1-4: de à I'hôpital d'urgence à consultation de routine, 1-5: de à l'hôpital d'urgence à autotraitement, 2-3: de consultation immédiate à visite chez le médecin dans les 24 heures, 2-5: de consultation de routine à autotraitement). En revanche, les corrections à la hausse les plus nombreuses concernaient la consultation de routine dans les 24 heures (3-4: consultation de routine à visite chez le médecin dans les 24 heures, 3-5: autotraitement à visite chez le médecin dans les 24 heures).

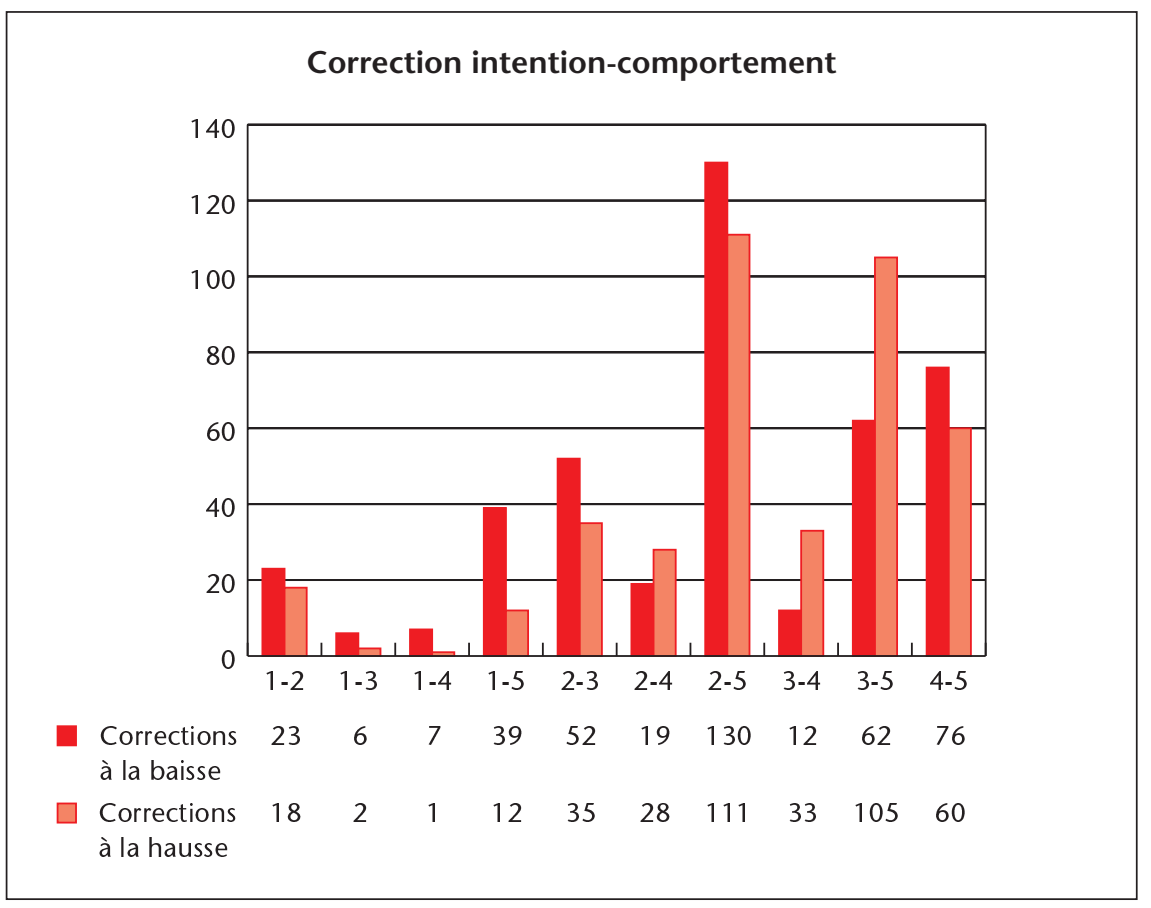

4 Lattimer V, Sassi F, Gorge S, Moore M, Turnbull J, Mullee M et al. Cost analysis of nurse telephone consultation in out of hours primary care: evidence from a randomised controlled trial. Br Med J 2000;320:1053-7. dation) de l'aiguillage au moyen d'études de cohorte, comme des tests de dépistage. L'IMSP continuera à évaluer scientifiquement les services de Medvantis medi-24 afin d'élaborer un processus global d'assurance qualité.

L'aiguillage étudié est également qualifié de «demand management». Il s'agit d'une forme d'encadrement du patient dont le but est de garantir que celui-ci bénéficie des meilleurs soins. La promotion d'une médecine peu coûteuse constitue l'une des motivations de ce projet, mais n'est pas sa première préoccupation.
Depuis l'introduction des centres d'expertise médicale, on a constaté le grand rôle qu'ils jouent dans la réduction des coûts de soins de santé. Cette étude n'est cependant pas conçue pour analyser les économies réalisées en la matière. C'est pourquoi les données récoltées ne feront l'objet que d'un bref débat à ce sujet.

Dans une optique économique, il est possible de distinguer des répercussions directes et indirectes. Les répercussions directes des différents niveaux d'aiguillage ne sont pas homogènes. En effet, une consultation en urgence à l'hôpital non fondée est nettement plus coûteuse qu'une consultation médicale ordinaire injustifiée chez le médecin de famille.

Dans l'ensemble, la comparaison entre l'intention avant l'entretien avec le centre d'expertise médicale et le comportement après celui-ci montre une nette correction à la baisse vers des niveaux de soins moins onéreux. Cette réduction touche essentiellement les soins d'urgence dispendieux (à l'hôpital d'urgence, consultation immédiate du médecin de famille ou de garde). Dans le cadre d'une étude randomisée, Lattimer et al. [4] ont démontré l'efficacité économique du télétriage pour les services d'urgence d'une coopérative médicale (55 généralistes / zone de 97000 habitants) dans le Wiltshire/UK. Les économies résultaient principalement d'une meilleure allocation des ressources des services d'urgence. Outre les réductions de coûts directes, nous supposons des économies indirectes bien plus importantes en raison de l'aiguillage correct et en temps utile des appelants vers un médecin. Les répercussions des services de Medvantis medi-24 sur les coûts de santé feront l'objet d'un autre travail de recherche.

\section{Remerciement}

Nous remercions vivement Madame Anne Sybil Götschi pour sa vigilante relecture et ses précieuses suggestions. 